\title{
Numerical Solution of Generalized Abel's Integral Equation by Variational Iteration Method
}

\author{
R. N. Prajapati ${ }^{1}$, Rakesh Mohan ${ }^{1}$, Pankaj Kumar ${ }^{2}$ \\ ${ }^{1}$ Department of Mathematics, Dehradun Institute of Technology, Dehradun, India \\ ${ }^{2}$ Kishori Lal Sharma Institue of Engineering \& Technology, Chandok, Bijnor, India \\ Email: narain.prajapati@gmail.com,rkmohan2k4@yahoo.com,Pankaj.rssb@gmail.com
}

Received June 1, 2012; revised September 13, 2012; accepted October 8, 2012

\begin{abstract}
In this paper, a user friendly algorithm based on the variational iteration method (VIM) is proposed to solve singular integral equations with generalized Abel's kernel. It is observed that an approximate solutions $y_{n}(x)$ converges to the exact solution irrespective of the initial choice $y_{0}(x)$. Illustrative numerical examples are given to demonstrate the efficiency and simplicity of the method in solving these types of singular integral equations.
\end{abstract}

Keywords: Variational Iteration Method; Singular Integral Equation; Abel's Kernel

\section{Introduction}

The real world problems in scientific fields such as solid state physics, plasma physics, fluid mechanics, chemical kinetics and mathematical biology are nonlinear in general when formulated as partial differential equations or integral equations. In the last two decades, many powerful techniques have been proposed to solve the singular integral equations and differential equation by using VIM [1-15].

The generalized Abel's integral equation of the second kind is given by

$$
y(x)=f(x)+\lambda \int_{0}^{x} \frac{y(t)}{(x-t)^{\alpha}} \mathrm{d} t, \quad 0 \leq x \leq 1,
$$

where $\lambda \in C$ is a parameter and $0<\alpha<1$.

Theorem 1. For each complex $\lambda \neq \infty$,

$$
y(x)=f(x)+\int_{0}^{x} R(x-t) f(t) \mathrm{d} t,
$$

is the unique solution of Equation (1),

where

$$
R(x)=\sum_{n=1}^{\infty} \frac{\left[\lambda \Gamma(1-\alpha) x^{(1-\alpha)}\right]^{n}}{x \Gamma[n(1-\alpha)]},
$$

The closed form solution (2) is not very useful in many cases where it is difficult to evaluate the integral appearing in (2). So, it is desirable to have numerical solution for the generalized Abel's integral Equation (1).

In the present paper, we have proposed an algorithm based on the variational iteration method to solve the generalized Abel's integral Equation (1). It is observed that the choice of the initial approximation $y_{0}(x)$ has a small effect on the efficiency of the method. The approximate solutions $y_{n}(x)$ will always converge to the exact solution.

\section{Basic Idea of Variational Iteration Method}

Variational Iteration method was first proposed by $\mathrm{He}$ [2-6] and has been successfully used by many researchers to solve various linear and nonlinear models [7-15]. The idea of the method is based on constructing a correction functional by a general Lagrange multiplier and the multiplier is chosen in such a way that its correction solution is improved with respect to the initial approximation or to the trial function.

Now, to illustrate the basic concept of the variational iteration method, we consider the following general nonlinear system:

$$
L[y(x)]+N[y(x)]=f(x),
$$

where $L$ is a linear operator, $N$ is a nonlinear operator and $f(x)$ is a known analytic function. The basic character of the method is to construct a correction functional for the system, which reads

$$
\begin{aligned}
& y_{n+1}(x)=y_{n}(x) \\
& +\int_{0}^{x} \lambda(s)\left[L y_{n}(s)+N \tilde{y}_{n}(s)-f(s)\right] \mathrm{d} s, \\
& n \geq 0
\end{aligned}
$$


where $\lambda$ is a general Lagrange multiplier, which can be identified optimally via variational theory, $y_{n}$ is the nth approximate solution, and $\tilde{y}_{n}$ is considered as a restricted variation, i.e. namely $\delta \tilde{y}_{n}=0$. Successive approximations, $y_{n+1}(x)$, will be obtained by applying the obtained Lagrange multiplier and a properly chosen initial approximation $y_{0}(x)$.

\section{Variational Iteration Method of Solution}

We consider the following iteration formula for Equation (1) in the following form

$$
y_{n+1}(x)=f(x)+\lambda \int_{0}^{x} \frac{y_{n}(t)}{(x-t)^{\alpha}} \mathrm{d} t, \quad n=0,1,2,3, \cdots ;
$$

where $y_{n}(x)$ is the nth approximate solution of (1) and $y_{0}(x)$ is an appropriately chosen initial guess. The value of $\lambda$ is found to be -1 .

\section{Numerical Examples}

The simplicity and accuracy of the proposed method are illustrated by the following numerical examples by computing the absolute error $E_{n}(x)=\left|y(x)-y_{n}(x)\right|$, where $y(x)$ is the exact solution and $y_{n}(x)$ is the nth approximate solution of the problem. The absolute error $E_{n}(x)$ has evaluated with examples 1 and 2 for value of $n=20$, and also the absolute error has calculated with examples 3 and 4 for different values of $n=30$ and $n=14$ respectively.

Example 1. Consider the following generalized Abel's integral equation of second kind

$$
\begin{aligned}
& y(x)=\text { FresnelC }(x) \\
& +\frac{16}{21} x^{7 / 4}{ }_{3} F_{4}\left[\left\{\frac{1}{4}, \frac{3}{4}, 1\right\},\left\{\frac{11}{16}, \frac{15}{16}, \frac{19}{16}, \frac{23}{16}\right\},-\frac{\pi^{2} x^{4}}{16}\right] \\
& -\int_{0}^{x} \frac{y(t)}{(x-t)^{1 / 2}} \mathrm{~d} t
\end{aligned}
$$

where FresnelC $(x)=\int_{0}^{x} \cos \left(\frac{\pi t^{2}}{2}\right) \mathrm{d} t$, with exact solution $y(x)=$ FresnelC $(x)$ solve the above integral equation by taking 4 different choices of the initial guess $y_{0}(x)$. It is observed that the method always converges to the exact solution.

Case 1a. Taking the initial guess $y_{0}(x)$. as

$$
\begin{aligned}
& y_{0}(x)=\text { Fresnel } C(x) \\
& +\frac{16}{21} x^{7 / 4}{ }_{3} F_{4}\left[\left\{\frac{1}{4}, \frac{3}{4}, 1\right\},\left\{\frac{11}{16}, \frac{15}{16}, \frac{19}{16}, \frac{23}{16}\right\},-\frac{\pi^{2} x^{4}}{16}\right],
\end{aligned}
$$

the various approximate solutions $y_{n}(x)$, obtained from Equation (6), are given as

$$
\begin{aligned}
& y_{1}(x)=\operatorname{FresnelC}(x) \\
& -\frac{8\left(\Gamma\left(\frac{3}{4}\right)\right)^{2} x^{5 / 2}}{15 \sqrt{\pi}}{ }_{3} F_{4}\left[\left\{\frac{1}{4}, \frac{3}{4}, 1\right\},\left\{\frac{7}{8}, \frac{9}{8}, \frac{11}{8}, \frac{13}{8}\right\},-\frac{\pi^{2} x^{4}}{16}\right]
\end{aligned}
$$

$$
y_{2}(x)=\operatorname{Fresnel} C(x)
$$$$
+\frac{\left(\Gamma\left(\frac{3}{4}\right)\right)^{3} x^{13 / 4}}{\Gamma\left(\frac{17}{4}\right)}{ }_{3} F_{4}\left[\left\{\frac{1}{4}, \frac{3}{4}, 1\right\},\left\{\frac{17}{16}, \frac{21}{16}, \frac{25}{16}, \frac{29}{16}\right\},-\frac{\pi^{2} x^{4}}{16}\right]
$$

Case 1b. Now we take a different value of the initial guess $y_{0}(x)=\operatorname{erfc}(x)$ and we get

$$
\begin{aligned}
& y_{1}(x)=\text { FresnelC }(x)-\frac{4 x^{3 / 4}}{3} \\
& +\frac{32 x^{7 / 4}}{21 \sqrt{\pi}}{ }_{2} F_{2}\left[\left\{\frac{1}{2}, 1\right\},\left\{\frac{11}{8}, \frac{15}{8}\right\},-x^{2}\right] \\
& +\frac{16}{21} x^{7 / 4}{ }_{3} F_{4}\left[\left\{\frac{1}{4}, \frac{3}{4}, 1\right\},\left\{\frac{11}{16}, \frac{15}{16}, \frac{19}{16}, \frac{23}{16}\right\},-\frac{\pi^{2} x^{4}}{16}\right], \\
& y_{2}(x)=\text { FresnelC }(x)+\frac{4\left(\Gamma\left(\frac{3}{4}\right)\right)^{2} x^{3 / 2}}{3 \sqrt{\pi}} \\
& 16\left(\Gamma\left(\frac{3}{4}\right)\right)^{2} x^{5 / 2} \\
& -\frac{15 \pi}{15} F_{2}\left[\left\{\frac{1}{2}, 1\right\},\left\{\frac{7}{4}, \frac{9}{4}\right\},-x^{2}\right] \\
& 8\left(\Gamma\left(\frac{3}{4}\right)\right)^{2} x^{5 / 2} \\
& -\frac{15 \sqrt{\pi}}{3} F_{4}\left[\left\{\frac{1}{4}, \frac{3}{4}, 1\right\},\left\{\frac{7}{8}, \frac{9}{8}, \frac{11}{8}, \frac{13}{8}\right\},-\frac{\pi^{2} x^{4}}{16}\right]
\end{aligned}
$$

Case 1c. Taking a different value of the initial guess $y_{0}(x)=x+1$ and solving we obtain

$$
\begin{aligned}
& y_{1}(x)=\text { FresnelC }(x)-\frac{4 x^{3 / 4}}{3}-\frac{16}{21} x^{7 / 4} \\
& +\frac{16}{21} x^{7 / 4}{ }_{3} F_{4}\left[\left\{\frac{1}{4}, \frac{3}{4}, 1\right\},\left\{\frac{11}{16}, \frac{15}{16}, \frac{19}{16}, \frac{23}{16}\right\},-\frac{\pi^{2} x^{4}}{16}\right], \\
& y_{2}(x)=\text { FresnelC }(x)+\frac{4\left(\Gamma\left(\frac{3}{4}\right)\right)^{2} x^{3 / 2}}{15 \sqrt{\pi}} \\
& \cdot\left[5+2 x-2 x_{3} F_{4}\left[\left\{\frac{1}{4}, \frac{3}{4}, 1\right\},\left\{\frac{7}{8}, \frac{9}{8}, \frac{11}{8}, \frac{13}{8}\right\},-\frac{\pi^{2} x^{4}}{16}\right]\right],
\end{aligned}
$$

Case 1d. In this case, we are taking the initial guess $y_{0}(x)=\cos x$ and using Equation (6) we get the following approximations of the solution 


$$
\begin{aligned}
& y_{1}(x)=\operatorname{Fresnel} C(x) \\
& -\frac{4 x^{3 / 4}}{3}{ }_{1} F_{2}\left[\{1\},\left\{\frac{7}{8}, \frac{11}{8}\right\},-\frac{x^{2}}{4}\right] \\
& +\frac{16}{21} x^{7 / 4}{ }_{3} F_{4}\left[\left\{\frac{1}{4}, \frac{3}{4}, 1\right\},\left\{\frac{11}{16}, \frac{15}{16}, \frac{19}{16}, \frac{23}{16}\right\},-\frac{\pi^{2} x^{4}}{16}\right] \text {, } \\
& y_{2}(x)=\operatorname{Fresnel} C(x) \\
& +\frac{4\left(\Gamma\left(\frac{3}{4}\right)\right)^{2} x^{3 / 2}}{3 \sqrt{\pi}}{ }_{1} F_{2}\left[\{1\},\left\{\frac{5}{4}, \frac{7}{4}\right\},-\frac{x^{2}}{4}\right] \\
& -\frac{8\left(\Gamma\left(\frac{3}{4}\right)\right)^{2} x^{5 / 2}}{15 \sqrt{\pi}}{ }_{3} F_{4}\left[\left\{\frac{1}{4}, \frac{3}{4}, 1\right\},\left\{\frac{7}{8}, \frac{9}{8}, \frac{11}{8}, \frac{13}{8}\right\},-\frac{\pi^{2} x^{4}}{16}\right]
\end{aligned}
$$

Figures 1-4 show the errors between the exact solution $y(x)$ and the approximate solutions $y_{20}(x)$ for the different initials choices of $y_{0}(x)$ for the above four cases.

\section{Conclusion}

In this paper, the variational iteration method has been successfully used to obtain a user friendly algorithm which is stable under the variations of initial guess $y_{0}(x)$ to solve the generalized Abel's integral equations. The variational iteration method yields solutions in the forms of a convergent series with easily calculable terms.

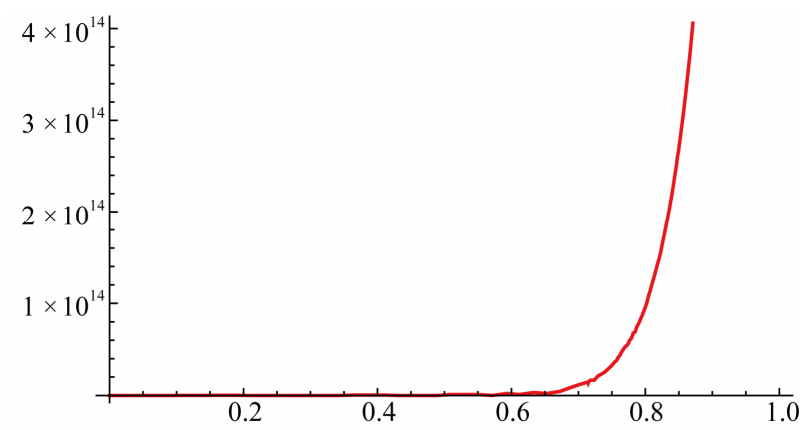

Figure 1. The absolute error $E(x)$ for example 1 (case a).

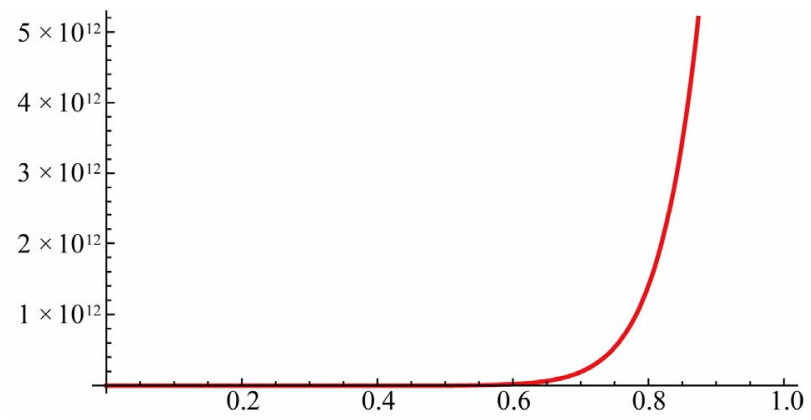

Figure 2. The absolute error $E(x)$ for example 1 (case $b)$.

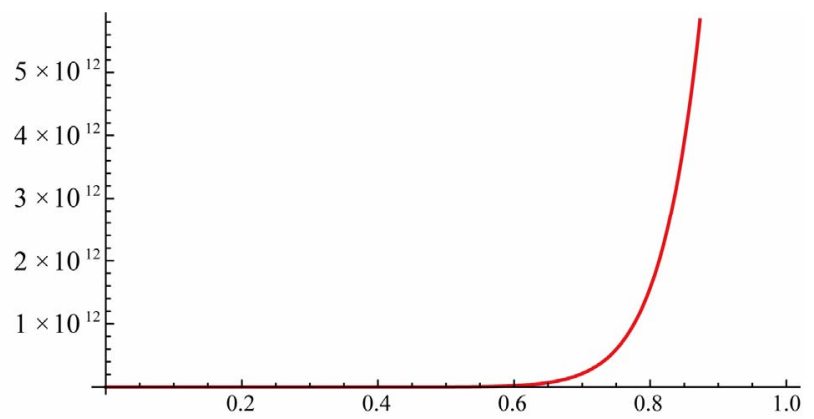

Figure 3. The absolute error $E(x)$ for example 1 (case c).

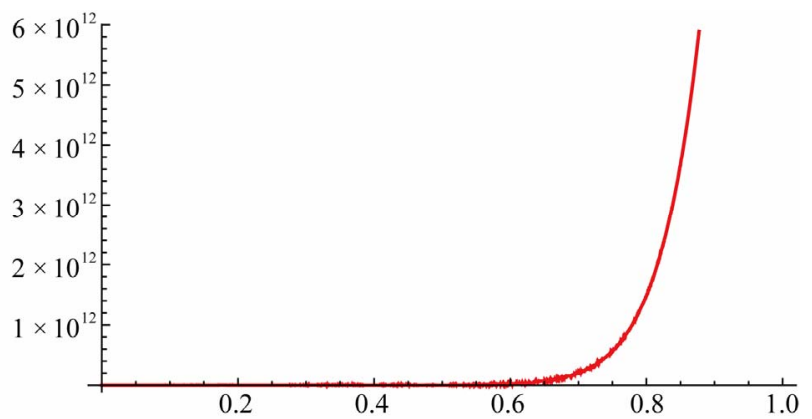

Figure 4. The absolute error for $E(x)$ example 1 (case d).

It is shown that the variational iteration method is a promising tool for such types of singular integral equations.

\section{REFERENCES}

[1] S. Abbasbandy and E Shivanian, "Applications Variational Iteration Method for n-th Order Integro-Differential Equations," Zeitschrift für Naturforschung A, Vol. 64a, 2009, pp. 439-444.

[2] J. H. He, "Variational Iteration Method-A Kind of Nonlinear Analytical Technique: Some Examples," International Journal of Non-Linear Mechanics, Vol. 34, No. 4, 1999, pp. 699-708. doi:10.1016/S0020-7462(98)00048-1

[3] J. H. He, "Some Asymptotic Methods for Strongly Non-Linear Equations," International Journal of Modern Physics B, Vol. 20, No. 10, 2006, pp. 1141-1199. doi:10.1142/S0217979206033796

[4] J. H. He, "Variational Iteration Method-Some Recent Results and New Interpretations," Journal of Computational and Applied Mathematics, Vol. 27, No. 1, 2007, pp. 3-17. doi:10.1016/j.cam.2006.07.009

[5] J. H. He, "Non-Perturbative Methods for Strongly Nonlinear Problems, Dissertation," Deverlag im Internet GmbH, Berlin, 2006.

[6] J. H. He and X. H. Wu, "Construction of Solitary Solution and Compton-Like Solution by Variational Iteration Method," Chaos, Solitons and Fractals, Vol. 29, 2006, pp. 108-113.

[7] S. A. Yousefi, A. Lotfi and M. Dehgan, "He's Variational 
Iteration Method for Solving Nonlinear Mixed VolterraFredholm Integral Equations," Computers \& Mathematics with Applications, Vol. 58, No. 11-12, 2009, pp. 2172-2176. doi:10.1016/j.camwa. 2009.03.083

[8] M. Tatari and M. Dehghan, “Improvement of He's Variational Iteration Method for Solving Systems of Differential Equations," Computers \& Mathematics with Applications, Vol. 58, No. 11-12, 2009, pp. 2160-2166. doi:10.1016/jcamwa. 2009.03.081

[9] R. Saadati, M. Dehghan, S. M. Vaezpour and M. Saravi, "The Convergence of He's Variational Iteration Method for Solving Integral Equations," Computers \& Mathematics with Applications, Vol. 58, No. 11-12, 2009, pp. 2167-2171. doi:10.1016/j.camwa.2009.03.008

[10] T. Ozis and A. Yildirim, "A Study of Nonlinear Oscillators with $u^{1 / 3}$ Force by He's Variational Iteration Method," Journal of Sound and Vibration, Vol. 306, No. 1-2, 2007, pp. 372-376. doi:10.1016/j.jsv.2007.05.021

[11] S. Momani and Z. M. Odibat, "Numerical Comparison of Methods for Solving Linear Differential Equations of Fractional Order," Chaos, Solitons and Fractals, Vol. 31,
No. 5, 2007, pp. $1248-1255$. doi:10.1016/j.chaos.2005.10.068

[12] S. Abbasbandy and E. Shivanian, "Application of Variational Iteration Method for nth-Order Integro-Differential Equations," Zeitschrift für Naturforschung A, Vol. 64a, 2009, pp. 439-444.

[13] S. Abbasbandy, "An Approximation Solution of a Nonlinear Equation with Riemann-Liouville's Fractional Derivatives by He's Variational Iteration Method," Journal of Computational and Applied Mathematics, Vol. 207, No. 1, 2007, pp. 53-58. doi:10.1016/j.cam.2006.07.011

[14] S. Abbasbandy, “A New Application of He's Variational Iteration Method for Quadratic Riccati Differential Equation by Adomian Polynomials," Journal of Computational and Applied Mathematics, Vol. 207, No. 1, 2007, pp. 59-63. doi:10.1016/j.cam.2006.07.012

[15] N. H. Sweilam and M. M. khader, "Variational Iteration Method for One Dimensional Nonlinear Thermo Elasticity," Chaos, Solitons and Fractals, Vol. 32, No. 1, 2007, pp. 145-149. doi:10.1016/j.chaos.2005.11.028 